\title{
Interband Tunneling Description of Holes in Wurtzite GaN at High Electric Fields
}

\author{
M. Hjelm, A. Martinez ${ }^{*}$, H.-E. Nilsson, and U. Lindefelt \\ Department of Information Technology and Media, Mid Sweden University, SE 85170 Sundsvall, Sweden \\ * Department of Electronics and Electrical Engineering, University of Glasgow, Glasgow G12 8LT, UK \\ e-mail: mats.hjelm@miun.se
}

\section{INTRODUCTION}

For semiconductors with hexagonal crystal geometry, like $4 \mathrm{H}$ and $6 \mathrm{H}-\mathrm{SiC}$, as well as $\mathrm{GaN}$, the number of atoms in the unit cell is relatively large, resulting in complicated band structures with a large number of bands. Due to this large number of bands, there are several regions in the Brillouin zone with small energy distances between the bands. The small differences make it necessary to consider band-to-band tunneling (interband transitions) in studies of charge transport at high and even at moderately high fields [1]. This implies that a multi-band description of the quantum state of the charge carrier has to be used. Monte Carlo (MC) simulations of $4 \mathrm{H}$-SiC show a considerable increase of the impact ionization coefficients compared with simulations not considering tunneling [2]. However, the interference in the carrier velocity has not been considered for an ensemble. This study investigates the effect of interference on hole velocity in wurtzite GaN using a density matrix approach.

\section{FUNDAMENTAL EQUATIONS}

The Krieger-Iafrate (KI) equations, which can be derived from the single particle Schrödinger equation in the presence of an electric field, can be written as $[1,3,4,5]$ :

$$
\begin{aligned}
-i \hbar \frac{\partial C_{n}^{\mathbf{k}_{0}}}{\partial t} & \\
= & \varepsilon_{n}[\mathbf{k}(t)] C_{n}^{\mathbf{k}_{0}}(t)-\sum_{n^{\prime}} q \mathbf{E} \cdot \mathbf{X}_{n n^{\prime}}[\mathbf{k}(t)] C_{n}^{\mathbf{k}_{0}}(t),
\end{aligned}
$$

where $C_{n}^{\mathbf{k}_{0}}$ is the probability amplitude that the a particle starting with the initial wave vector $\mathbf{k}_{0}$, with wave vector $\mathbf{k}(t)(\dot{\mathbf{k}}(t)=q \mathbf{E} / \hbar)$, and $\varepsilon_{n}$ the energy in band $n . \mathbf{X}_{n n^{\prime}}$ is the coupling between the bands $n$ and $n$ ' and is given by:

$$
\mathbf{X}_{n n^{\prime}}[\mathbf{k}(t)]=-\frac{i}{\Omega_{\text {cell }}} \int_{\text {cell }} u_{n}^{*}[\mathbf{k}(t), \mathbf{r}]_{\mathbf{k}} u_{n}[\mathbf{k}(t), \mathbf{r}] d^{3} r
$$

where $u_{n}[\mathbf{k}(t), \mathbf{r}]$ is the periodic part of the Bloch function and the integration is performed over one unit cell with the volume $\Omega_{\text {cell }}$.

The expectation value of the carrier velocity is given by:

$$
\begin{aligned}
\mathbf{V}_{\mathbf{k}_{0}}(t) & =\sum_{n}\left|C_{n}^{\mathbf{k}_{0}}(t)\right|^{2} \frac{1}{\hbar} \nabla_{\mathbf{k}(t)}[\mathbf{k}(t)] \\
& +\sum_{n>n^{\prime}} \sum_{n^{\prime}} \frac{2 \hbar}{m_{0}} \operatorname{Im}\left(C_{n^{\prime}}^{\mathbf{k}_{0} *} C_{n}^{\mathbf{k}_{0}}\right) \\
& \times \int_{\text {cell }} u_{n^{\prime}}^{*}[\mathbf{k}(t), \mathbf{r}] \nabla u_{n}[\mathbf{k}(t), \mathbf{r}] d^{3} r .
\end{aligned}
$$

Equation (2) determines the trajectory of the Bloch charge carriers during the free flight between the scattering events. The first term is a mean value of the group velocity while the second term, which we call the interference term, is a quantum correction depending on the relative phase of the expansion coefficients $C_{n}^{\mathbf{k}_{0}}$.

\section{SIMULATIONS AND RESULTS}

In Fig. 1 the band structure for the uppermost 6 valence bands of $\mathrm{GaN}$ is shown for a segment parallel to the $Г A$ direction (crystal axis) starting at a point in the $Г М K$ plane. The $\mathrm{x}$-axis scale corresponds to an electric field of $0.4 \mathrm{MV} / \mathrm{cm}$. This field is applied (parallel to $\Gamma$ A direction) to the ensemble of holes in equilibrium. The carriers move and tunnel between different bands, which is numerically calculated according to Eq. 1. From the resulting probabilities the average energy and velocity of the 
ensemble is calculated. We have considered the cases of two field strengths 0.4 and $4 \mathrm{MV} / \mathrm{cm}$.

Fig. 2 shows the mean energy of the ensemble, both with and without tunneling. After studying Fig. 1 , it may be believed that the holes tunnel from band 3 to band 4 , but the contrary is actually the case, which is shown in Fig. 3. The increase of energy shown in Fig. 2 is explained by tunneling from band 1 to 2, which is more important because of the larger number of holes initially in band 1. In Fig. 4 the expectation value of the velocity is shown calculated according to Eq. 3, the classical velocity (Eq. 3 except interference), and the band velocity, i.e. the holes are assumed to stay in the same band.

\section{CONCLUSIONS}

In this work we present average energy calculated with the KI equation, i.e. considering band-toband tunnelling, for an ensemble of holes and compare it with the energy without tunnelling. Additionally, the velocity is calculated considering interference and compared with the velocity not considering interference and without tunnelling. At the workshop results will be shown for $4 \mathrm{MV} / \mathrm{cm}$ and a density matrix formulation of the KI equations will be given.

\section{REFERENCES}

[1] A. Martinez, U. Lindefelt, and H-E. Nilsson, J. Appl. Phys. 93, 9784 (2003).

[2] H-E. Nilsson, A. Martinez, U. Sannemo, M. Hjelm, E. Bellotti, and K. Brennan, Physica B 31468 (2002).

[4] J.B. Krieger and G. J. Iafrate, Phys. B 33, 5494 (1986).

[5] L. V. Keldysh, Sov. Phys. JEPT 6, 763 (1958).

[6] J. Rotvig et al., Phys. Rev. B 54, 17961 (1996).

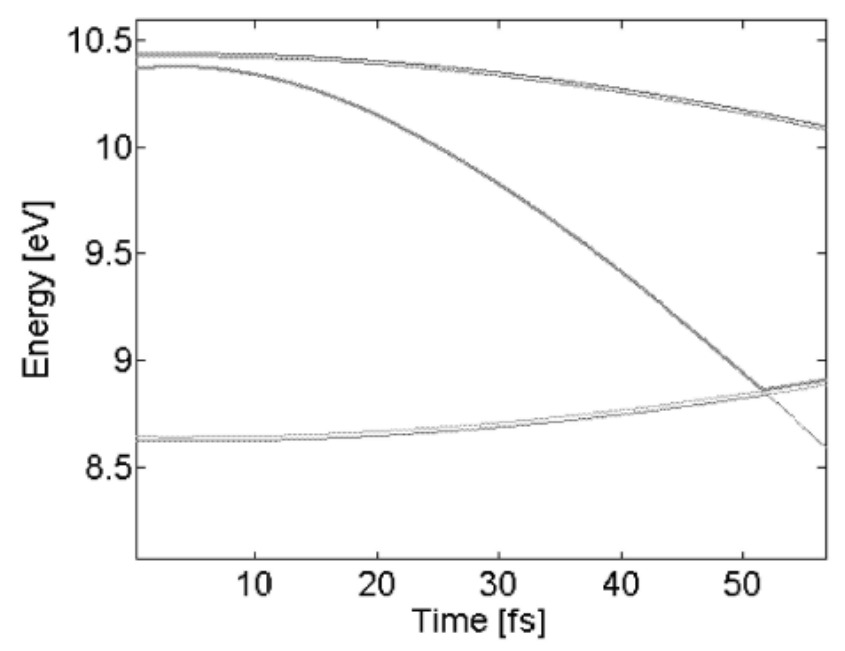

Fig. 1. Band structure in the $\Gamma A$ direction for wurtzite $\mathrm{GaN}$, $\mathrm{E}=0.4 \mathrm{MV} / \mathrm{cm}$.

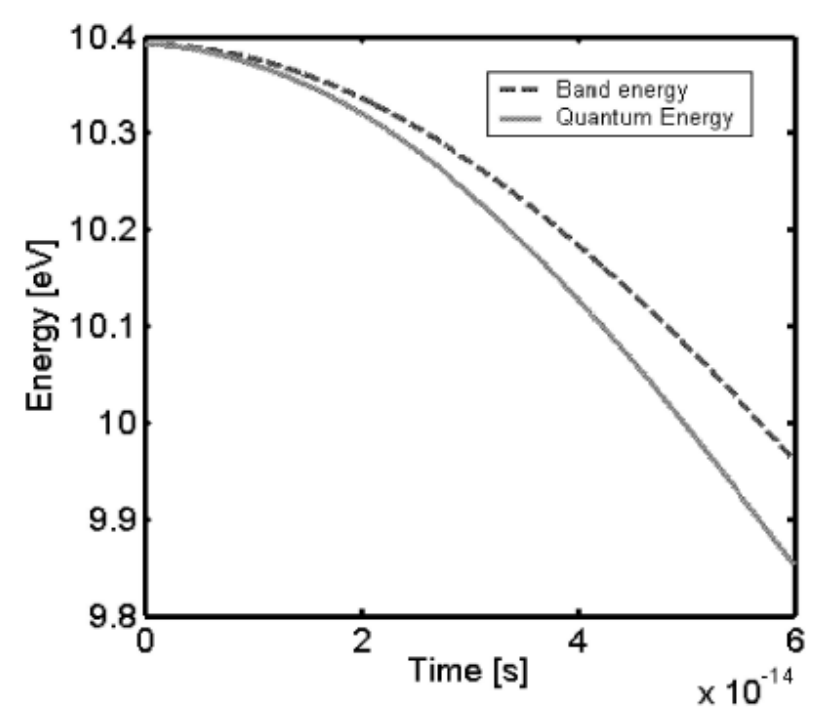

Fig. 2. Quantum energy (tunneling considered) and band energy (no tunneling) of the ensemble for $0.4 \mathrm{MV} / \mathrm{cm}$.

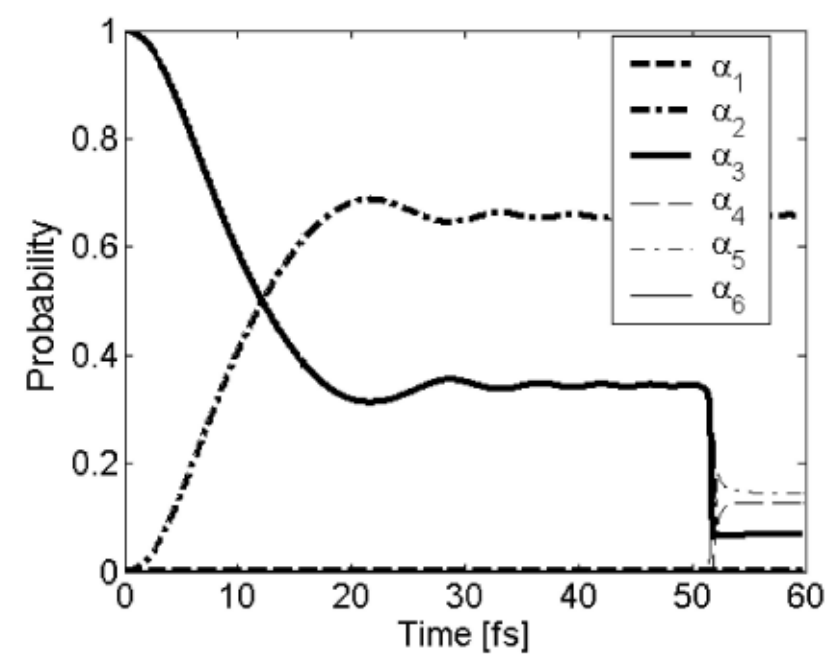

Fig. 3. The probability distribution between bands for a hole initially in the third band for $0.4 \mathrm{MV} / \mathrm{cm}$.

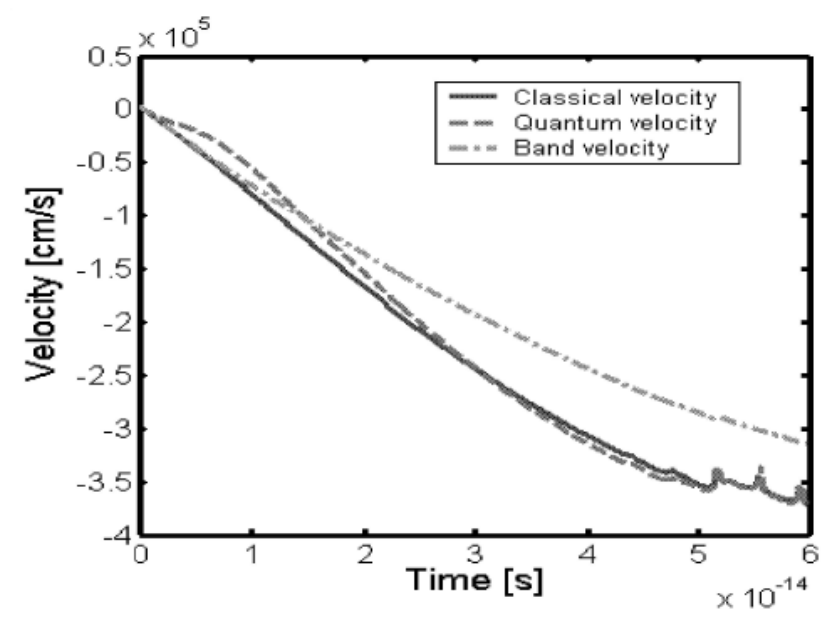

Fig. 4. Simulated velocity considering tunneling including interference (quantum velocity), tunneling not including interference (classical velocity), and without tunneling (band velocity). 